\title{
HIPERPLASIA NODULAR HISTIOCÍTICA: UMA MANIFESTAÇÃO RARA À EXPOSIÇÃO AO ASBESTO
}

\author{
NODULAR HISTIOCYTIC HYPERPLASIA: A RARE MANIFESTATION OF ASBESTO EXPOSURE
}

Layni Storch ${ }^{1 *}$, Lívia Marques da Silva Gama ${ }^{2}$, Fernando Guimarães Porto ${ }^{3}$, Isabella Vargas Baldon ${ }^{4}$, André

Felipe Candeas Amorim ${ }^{5}$, Francyellen Bandeira dos Santos ${ }^{6}$, Tony Coimbra Clara.

${ }^{1}$ Médica Residente de Gastroenterologia do Hospital Universitário Cassiano Antônio de Moraes.

${ }^{2}$ Médica Pneumologista do Hospital Universitário Cassiano Antônio de Moraes.

${ }^{3}$ Médico Residente de Clínica Médica do Hospital Universitário Cassiano Antônio de Moraes.

${ }^{4}$ Acadêmica de Medicina da Universidade Federal do Espírito Santo.

${ }^{5}$ Acadêmico de Medicina da Universidade Federal do Espírito Santo.

${ }^{6}$ Acadêmica de Medicina da Universidade Federal do Espírito Santo.

${ }^{7}$ Médico pela Escola Superior de Ciências da Santa Casa de Misericórdia de Vitória.

* Layni Storch. Avenida Comandante Álvaro Martins, 71- Mata da Praia. Vitoria- ES. CEP:29066- 050 cel : 027- 999355107. Email: laystorch@gmail.com

Instituição: Hospital Universitário Cassiano Antônio de Moraes. Av. Marechal Campos, 1355, Santa Cecília Vitória - ES - 29043-260

\section{RESUMO}

A hiperplasia nodular histiocítica $(\mathrm{HNH})$ consiste em uma condição rara, sem predileção por sexo ou idade, marcada por um processo proliferativo benigno, por vezes confundido com neoplasia maligna, constituído majoritariamente por histiócitos. Inflamação crônica local e irritação mecânica estão entre as principais causas postuladas para essa entidade. Patologias malignas, por outro lado, representam os principais diagnósticos diferenciais, o que evidencia a importância clínica de seu diagnóstico assertivo sendo a imunohistoquímica uma importante ferramenta diagnóstica. Nesse artigo, os autores descrevem um relato de caso de hiperplasia nodular histiocítica pleuro-pulmonar em um paciente do sexo masculino, não tabagista, com história de exposição ao asbesto o qual provavelmente incitou um processo inflamatório crônico com proliferação de histiócitos. $O$ paciente apresentava dispnéia, dor pleurítica e hemoptise além de derrame pleural hemorrágico recorrente evoluindo a óbito por exacerbação dos sintomas. Apesar da entidade ser descrita na literatura como uma proliferação celular benigna histiocitária, há raros casos relatados sobre a evolução e tratamento da doença.

Palavras chaves: Hiperplasia nodular histiocítica, asbesto, pleura, pulmão.

\section{ABSTRACT}

A nodular hyperplasia histiocytic $(\mathrm{HNH})$ is a rare condition, with no preference for gender or age, marked by a benign proliferative process, sometimes confused with malignant neoplasia, consisting mostly of histiocytes. Local chronic inflammation and mechanical irritation are among the main causes postulated for this entity. Malignant pathologies, on the other hand, represent the main differential diagnoses, or that highlight the clinical importance of their assertive diagnosis, being an immunohistochemistry an important diagnostic tool. In this article, the authors describe a case of pulmonary pulmonary histiocytic hyperplasia in a non-smoking male patient with a history of asbestos exposure or the likelihood of inciting a chronic inflammatory process with histiocytes proliferation. The patient has dyspnea, pleuritic pain and hemoptysis and recurrent hemorrhagic pleural effusion following death due to exacerbation of symptoms. Although the entity is used in the literature as a benign histiocytic cell proliferation, there are rare cases related to the evolution and treatment of the disease.

Key words: Histiocytic nodular hyperplasia, asbestos, pleura, lung. 


\section{INTRODUÇÃO}

A hiperplasia nodular histiocítica, anteriormente conhecida como Hiperplasia nodular histiocítica/ mesotelial, é uma condição benigna rara, descrita em 1975 como pequenos nódulos de células redondas, de citoplasma acidófilo, inicialmente consideradas de origem mesotelial (). Posteriormente, foi evidenciada a presença marcante de agregados de histiócitos (, ). Um saco herniário foi o primeiro sítio descrito da doença e desde então foi vista em outros locais como pericárdio, coração, pleura, pulmões, cavidade pélvica e bexiga (). Consiste na proliferação reativa predominante de histiócitos e dispersas células mesoteliais, secundárias à irritação/ inflamação crônica. A proliferação celular histiocitária com imunorreatividade difusa positiva para o marcador histiocítico CD68, corrobora o diagnóstico ${ }^{1,5}$. Dentre os diagnósticos diferenciais, destacam-se lesões mesoteliais primárias e neoplasias malignas como mesoteliomas, adenocarcinomas, tumores de células granulosas ou histiocitose de células de Langerhans (,). Nesse contexto, a imunohistoquímica se apresenta como uma importante ferramenta diagnóstica para diferenciar essas patologias, uma vez que a presença de citoqueratinas e ausência de positividade CD68 associado à citologia com atipias celulares, reforça à malignidade (). A seguir, descrevemos um caso de Hiperplasia nodular histiocítica pleural e pulmonar em um paciente com exposição ocupacional ao asbesto e queixa de dor pleurítica.

\section{OBJETIVO}

Descrever um caso clínico de hiperplasia nodular histiocítica pulmonar e pleural em um paciente submetido à exposição crônica por asbesto.

\section{DESCRIÇÃO}

J.D, masculino, 53 anos, diabético não insulino-dependente, não tabagista, trabalhador de lavoura de café, com exposição ocupacional crônica a asbesto em construção civil. Paciente com história de dor pleurítica à direita e dispnéia aos grandes esforços (MRC:0), desde 2012, evoluiu em abril de 2017 com piora dos sintomas e volumoso derrame pleural à direita. Sem relato de febre ou emagrecimento nesse período.

Tomografia computadorizada (TC) de tórax demonstrou atelectasia compressiva, condensação do parênquima pulmonar adjacente e diminutos nódulos esparsos no pulmão esquerdo. Exame micológico direto para pesquisa de bacilo álcoolácido resistente e cultura de líquido pleural negativos. Sorologias para HIV, hepatites B e C, sífilis e anticorpos para pesquisa de doenças reumatológicas também negativos. Realizada biópsia pleural direita por videotoracoscopia que evidenciou infiltrados histiocitários sem sinais de malignidade.

Durante o ano de 2017, sintomas recorrentes levaram o paciente a buscar o pronto atendimento em diversas ocasiões, sendo decidido pela realização de nova biópsia pleural, a qual manteve o mesmo padrão histológico descrito anteriormente. Nesse período, pleurectomia, pleurodese e toracotomia com drenagem à direita foram necessárias devido à volumosa coleção pleural hemática de contornos lobulados, compatível com derrame pleural hemorrágico.

Paciente evoluiu com hemoptise, perda de 9 kg em 4 meses e piora da dispneia (MRC: 2) em dezembro de 2017, além de derrame pleural hemorrágico recorrente. A espirometria revelou distúrbio ventilatório misto acentuado e nova tomografia computadorizada de tórax demonstrou doença infiltrativa pleural direita com aumento do acometimento pulmonar difuso e bilateral por múltiplas lesões nodulares. Notou-se ainda redução volumétrica do pulmão direito por encarceramento pela extensa doença pleural infiltrativa, com aumento do derrame pleural deste lado (figuras $1 \mathrm{e}$ 2).

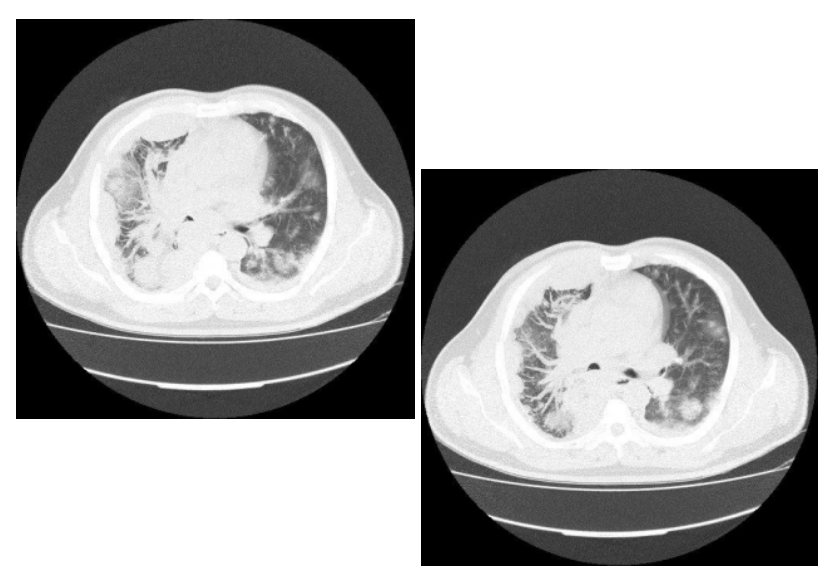

Figuras 1 e 2: Tomografia de tórax evidenciando doença infiltrativa pleural no hemitórax direito, com áreas de espessamento pleural irregular, de aspecto sólido, formando volumosas massas, principalmente junto a pleura mediastinal, ocasionando encarceramento pulmonar. Nota-se ainda lesões nodulares pulmonares e espessamento regular dos septos interlobulares no pulmão direito associado à líquido livre na cavidade pleural. 
Nova videobroncoscopia mostrou redução do calibre de brônquios do lobo médio e inferior direito, sendo a pesquisa de células neoplásicas negativa no lavado broncoalveolar. Dessa forma, optou-se por proceder com nova biópsia pleuropulmonar guiada por TC, na qual o histopatológico revelou parênquima pulmonar e pleural sem atipias celulares, porém com agregados de histiócitos xantomatosos (figuras 3 e 4). A imunohistoquímica (IHQ) apresentou positividade para CD68 e negatividade para pancitoceratinas (AE1/AE3), afastando a possibilidade de malignidade (figuras 5 e6).

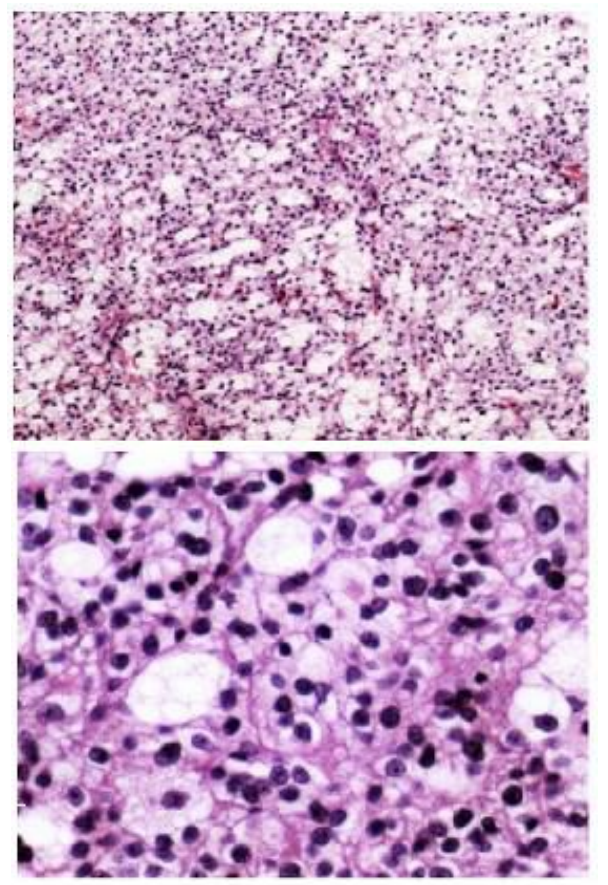

Figuras 3 e 4: Cortes histológicos de parênquima pulmonar e pleural direito evidenciando processo inflamatório crônico rico em histiócitos xantomatosos.

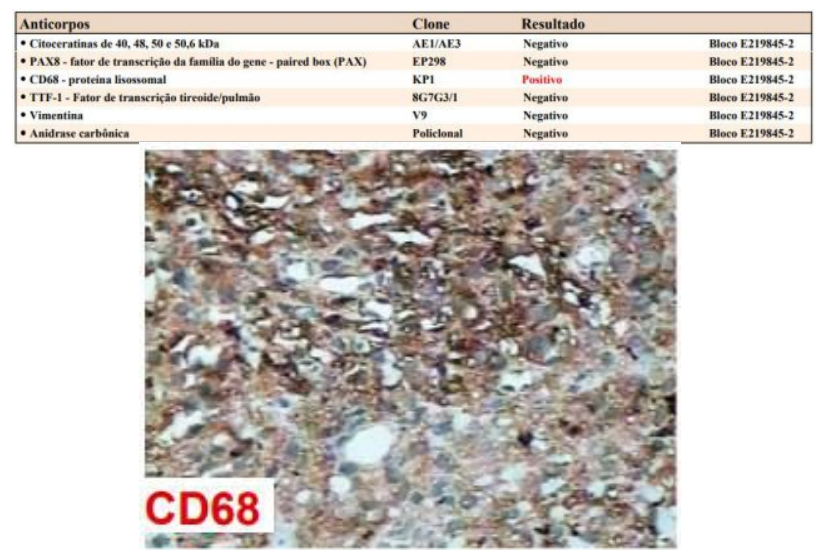

Figuras 5 e 6: Cortes histológicos com análise imunohistoquímica de parênquima pulmonar e pleural direito evidenciando negatividade para pancitoceratina (AE1/AE3) desfavorecendo a possibilidade de carcinoma, mesotelioma e ratificando a positividade para CD68.
Em março de 2018, houve nova internação hospitalar devido piora dos sintomas pulmonares (MRC:4 e dor pleurítica mantida, sem hemoptise no momento) com evidências clínicas e laboratoriais de infecção pulmonar excluídas. Realizou tratamento com prednisona $60 \mathrm{mg} / \mathrm{dia}$, com redução dos episódios de tosse com hemoptise, porém sem alteração do padrão de dispneia e dor. Após 3 meses de uso de imunossupressor, foi a óbito por provável hemorragia alveolar/ hemoptise e insuficiência respiratória.

\section{DISCUSSÃO}

A Hiperplasia histiocítica é um processo proliferativo benigno, predominantemente composto por histiócitos com células mesoteliais dispersas no meio. Rosai e Dehner em 1975, antes do advento da imuno-histoquímica, observaram amostras de sacos herniários/ peritoneais com proliferação de células mesoteliais que simulava processo neoplásico e chamaram de hiperplasia mesotelial nodular. O mecanismo fisiopatológico envolvido no desenvolvimento desses nódulos reativos não foi discutido no artigo. Em 1997, Chan e cols. relataram 2 casos com alterações histológicas semelhantes vistas em biópsias transbrônquicas porém com células histiocitárias associadas e, cunharam o termo "hiperplasia histiocítica / mesotelial nodular" ${ }^{2},{ }^{6}, 7$.

Posteriormente, com o surgimento da imunohistoquímica, o primeiro relato de envolvimento pleural por esse processo reativo demonstrou que a proliferação representava uma população mista, porém, predominantemente de histiócitos CD68 positivos e dispersas células mesoteliais positivas para citoqueratina, sendo, portanto, denominado de hiperplasia nodular histiocítica (HNH).

A $\mathrm{HNH}$ pode ocorrer em qualquer sítio mesotelial e, antes do uso generalizado da imunohistoquímica, recebeu vários diagnósticos errôneos como carcinoma metastático e mesotelioma em amostras de sacos herniários. Todos os casos com etiologia comprovada descritos na literatura envolveram a fisiopatologia de irritação do revestimento mesotelial, seja por um processo físico, neoplásico, inflamatório ou infeccioso. As etiologias pulmonares subjacentes conhecidas e descritas nas literatura que favorecem o processo incluem, pneumonia, abscesso subfrênico, carcinoma de células escamosas pulmonares e 
pneumotórax espontâneo ${ }^{1},{ }^{14}$.

Perante o quadro do paciente relatado, sugere-se que as lesões pleuro- pulmonares foram decorrentes da longa exposição ao asbesto, com consequente inflamação crônica local e formação de aglomerados histiocitários circundados por linfócitos, neutrófilos e células plasmáticas. Devido às alterações tomográficas, principalmente espessamento irregular pleural, história ocupacional e clínica do paciente, as hipóteses diagnósticas aventadas iniciais incluíam asbestose e mesotelioma pleural, sendo o último de pior prognóstico. Porém, a negatividade para citoqueratinas afasta a possibilidade de lesões malignas, como por exemplo mesotelioma ou carcinoma. Já a presença de histiócitos com positividade para CD68 na imuno-histoquímica, fortalece o diagnóstico de hiperplasia nodular histiocítica.

O tratamento ideal, segundo opinião de especialistas, consiste na ressecção cirúrgica extensa ou transplante pulmonar. Entretanto, as lesões pulmonares difusas do paciente contraindicaram o procedimento cirúrgico/ transplante. Dessa forma, optou-se pela tentativa de corticoterapia (prednisona $60 \mathrm{mg} / \mathrm{dia}$ ) que foi mantida por 3 meses, observando-se melhora parcial dos sintomas, porém o paciente evoluiu a óbito.

\section{CONCLUSÃO}

A HNH causa uma hiperplasia benigna de células histiocitárias que podem comprometer a maioria dos locais que possuem células mesoteliais, como pericárdio, peritônio e tecido pleural, raramente comprometendo outros sítios(). Desse modo, é importante atentar-se ao diagnóstico diferencial, uma vez que pode ser facilmente confundida com lesões neoplásicas ou outras lesões benignas do sistema mesotelial. Ressalta-se ainda, o papel da imunohistoquímica com positividade ao CD68, um marcador de células histiocitárias, que auxilia no diagnóstico ao afastar lesões de origem puramente mesotelial (, ).

Nesse artigo, relatamos um caso de $\mathrm{HNH}$ pleuro-pulmonar para adicionar ao espectro de alterações histopatológicas que ocorrem na exposição ao asbesto sendo rara essa descrição na literatura bem como seu tratamento. Apesar de ser descrita histologicamente como uma lesão benigna, pode levar a sinais e sintomas que levam a óbito como no caso descrito acima.

\section{REFERÊNCIAS}

1. Ordonez NG, Ro JY, Ayala AG. Lesions described as nodular mesothelial hyperplasia are primarily composed of histiocytes. The American journal of surgical pathology. 1998;22(3):285-92.

2. Chan JK, Loo KT, Yau BK, Lam SY. Nodular histiocytic/mesothelial hyperplasia: a lesion potentially mistaken for a neoplasm in transbronchial biopsy. The American journal of surgical pathology. 1997;21(6):658-63.

3. Cabibi D, Lo lacono G, Raffaele F, Dioguardi S, Ingrao S, Pirrotta A, et al. Nodular histiocytic/mesothelial hyperplasia as consequence of chronic mesothelium irritation by subphrenic abscess. Future oncology (London, England). 2015;11(24 Suppl):51-5.

4. Rosai J, Dehner LP. Nodular mesothelial hyperplasia in hernia sacs: a benign reactive condition simulating a neoplastic process. Cancer. 1975;35(1):165-75.

5. Choi YL, Song SY. Cytologic clue of so-called nodular histiocytic hyperplasia of the pleura. Diagnostic cytopathology. 2001;24(4):256-9.

6. Suarez-Vilela D, Izquierdo-Garcia FM. Nodular histiocytic/mesothelial hyperplasia: a process mediated by adhesion molecules? Histopathology. 2002;40(3):299-300.

7. Chikkamuniyappa S, Herrick J, Jagirdar JS. Nodular histiocytic/mesothelial hyperplasia: a potential pitfall. Annals of diagnostic pathology. 2004;8(3):115-20.

8. Bejarano PA, Garcia MT, Ganjei-Azar P. Mesothelial cells in transbronchial biopsies: a rare complication with a potential for a diagnostic pitfall. The American journal of surgical pathology. 2007;31(6):914-8.

9. Rossi G, Cavazza A, Guicciardi N, Marchioni A. Nodular histiocytic/mesothelial hyperplasia on transthoracic biopsy: another source of potential pitfall in a lesion frequently present in spontaneous pneumothorax. Histopathology. 2008;52(2):250-2.

10. Cai Z, Xie Q, Wang X, Guo B, Wang X, Wang K. [Nodular histiocytic/mesothelial hyperplasia: a clinicopathologic analysis of 7 cases]. Zhonghua bing li xue za zhi = Chinese journal of pathology. 2014;43(4):256-9. 
11. Jiao N, Zhang W, Wang W, Wang X, Liu Y, Xu G, et al. Mesothelial/monocytic incidental cardiac excrescence: a case report and review of literature. International journal of clinical and experimental pathology. 2014;7(9):6219-24. 12. Chung Y, Abdul R, Jang SM, Choi JS, Jang K. Pelvic Nodular Histiocytic and Mesothelial Hyperplasia in a Patient with Endometriosis and Uterine Leiomyoma. Journal of pathology and translational medicine. 2016;50(5):397-400. 13. Rao S, Aggarwal R, Vijay C, Kumar A. Nodular histiocytic hyperplasia of pericardium: An uncommon lesion posing diagnostic challenge. Lung India : official organ of Indian Chest Society. 2014;31(2):198-200.

14. Merg K B, Liebling P D, Kubik M J, Attanoos R, et al. Pleural nodular mesothelial/ histiocytic hyperplasia associated wyth syphilis. Humam Pathology.Case reports. 2018;vol13- pages18-20. 